LAWRENCE LIVERMORE N A TION AL LABORATORY

\title{
Calculation of Phonon Density of States for Alpha-U
}

A.L. Kutepov

April 16, 2004 
This document was prepared as an account of work sponsored by an agency of the United States Government. Neither the United States Government nor the University of California nor any of their employees, makes any warranty, express or implied, or assumes any legal liability or responsibility for the accuracy, completeness, or usefulness of any information, apparatus, product, or process disclosed, or represents that its use would not infringe privately owned rights. Reference herein to any specific commercial product, process, or service by trade name, trademark, manufacturer, or otherwise, does not necessarily constitute or imply its endorsement, recommendation, or favoring by the United States Government or the University of California. The views and opinions of authors expressed herein do not necessarily state or reflect those of the United States Government or the University of California, and shall not be used for advertising or product endorsement purposes.

This work was performed under the auspices of the U.S. Department of Energy by University of California, Lawrence Livermore National Laboratory under Contract W-7405-Eng-48. 


\section{RUSSIAN FEDERAL NUCLEAR CENTER \\ ALL-RUSSIAN SCIENTIFIC RESEARCH INSTITUTE OF TECHNICAL PHYSICS NAMED \\ AFTER ACADEMICIAN E.I. ZABABAKHIN}

\section{REPORT}

Task Order No. B530324 under Master Task Agreement No. B239614

"Quantum-Mechanical Simulation of Material Properties"

Deliverable 4. Task 8.

\section{CALCULATION OF PHONON DENSITY OF STATES FOR ALPHA-U}

Principal Investigator

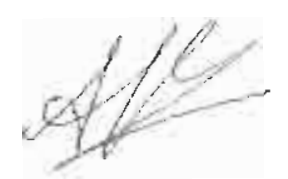

A. L. Kutepov 


\begin{abstract}
Phonon density of states for $\alpha$ - $U$ was calculated with the linear response theory and the RSPFLAPW (fully relativistic full potential spin-polarized linearized-augmented-plane-wave) method. It has becn used for studying the temperature dependance of the specific heat of uranium. It. was shown that the details of the phonon DOS are not very important for the specific heat, and practically the same results can be obtained with Debye model.
\end{abstract}




\section{INTRODUCTION}

Uranium, one of the heaviest clements discovered in the nature, has attracted considerable atterition to itself per the last decades, mainly due to its usag'e as reactor fuel and in nuclear

weapons. The interesting properties of this element include unusuct temperature dependance of elastic constants, ${ }^{1-3}$ availability of waves of charge density, ${ }^{4,5}$ anisotropic expansion at. heating up and, sinilarly to other light actinides, unique crystalline structure. ${ }^{6}$ Also, an interest in the phononic properties of $U$ has grown up last years. Despite the fact, that there are some problems in measuring the phonon dispersion for actinides, the lattice dynarnics studies on $\mathrm{U}$ have been performed at room temperature and below, ${ }^{7,8}$ motivated in part by the discovery of several charge density wave transitions at low temperatures. ${ }^{4,5}$ It has been found an unusually large thermal softening of phonon frequencies. Analysis conducted in ref. [8] has shown that this phonon softening originates with the softening of a harmonic solid, as opposed to vibrations in anharmonic potentials. Studying the phonon density of statcs (DOS) is also interesting because of the vibrational entropy contribution to the high temperature phase stability of the metal.

In the present work the phonon DOS of uranium has been calculated with linear response theory and full-potertial fully relativistic linear-augmented-plane-wave method. ${ }^{9}$ The basis functions in this method are especially well suit for describing such an open structures as $a-U$. The motivation for carrying out such a study was to calculate the phonon DOS of $\mathrm{U}$ theoretically and to compare the vibrational and electronic contributions in the thermodyramic functions of $\mathrm{U}$ at $\mathrm{P}=0$. This analysis is interesting because it has becn shown ${ }^{8}$ that these two contributions cannot be separated.

This paper is organized as follows. In section II the calculational parameters are provided. The results of studying are presented in section III.

\section{THE PARAMETERS OF CALCULATIONS}

The details of the calculations of the phonon DOS are the following: The dynamical matrix and the phonon energies $\omega_{\text {go }}$ ( $\alpha$ labels different phonon states at a given $q$ ) for a set of irraducible $q$ points at the $3 \times 3 \times 3$ reciprocal lattice grid have been calculated in accordance to the formulaes presented in previous report. ${ }^{10}$ After that, the phonon density 
of states has been found via the definition

$$
g(\omega)=\sum_{\mathrm{q} \alpha} \delta\left(\omega-\omega_{\mathrm{q} \alpha}\right)
$$

The integration over the Brilloum zone has been performed with the tetrahedron method. ${ }^{11}$ Relatively small number of $q$-points is related with the fact that self-consistent. calculation of dynamical matrix appeared to be very expensive. We think, however, that. this number of $q$-points is quite enough because the phonon dispersions are vell behaved functions of the wave vector.

The self consistent calculations for the first order electronic density have been performed in the local density approxination (LDA) because at the moment we have some problems in using the generalized gradient approximation (GGA) in linear response study (in that case the exchange-correlation potential already contains second derivatives of charge density and the differentiation of it brings some bad controlled errors in calculation).

The calculation of first order change in charge density for every wave vector $q$ involved the same parameters as in the previous studies on uranium. ${ }^{12}$ The radii of the muffintin spheres werc taken identical for all the atoms and the muffin-tin spheres were alniost touching. Within the muffin-tin spheres the charge density and potontial were expanded in spherical harmonics with a cutoff $L_{\max }$ equal to 6 . The angular momentum cutoff $L_{\max }=8$ was used for the basis functions. The basis set has also included the semicore orbitals $5 d, 6 s$ and $6 p$. The plane wave expansion of the wave functions was terminated in such a manner that the total energy was convorged better than $1 \mathrm{mRy}$ per atom in all the cases. The basis size was in the range $800-850$ (with a small dependence on the $\mathbf{k}$-point). Electronic density and effective potential in the interstitial were represented by Fourier series consisting of about 30000 plane waves. The integration ovor Brillouin zone (over the electronic states) has been carried out by the improved tctrahedron method ${ }^{11}$ with sampling $9 \times 9 \times 9$.

\section{PHONON DOS OF $\alpha$-U. TEMPERATURE DEPENDANCE OF SPECIFIC HEAT AT ZERO PRESSURE.}

The performing of this Task had been started with the calculation of the phononic density of states at a few volumes close to expcrimental equilibrium volume $V_{0}$. 


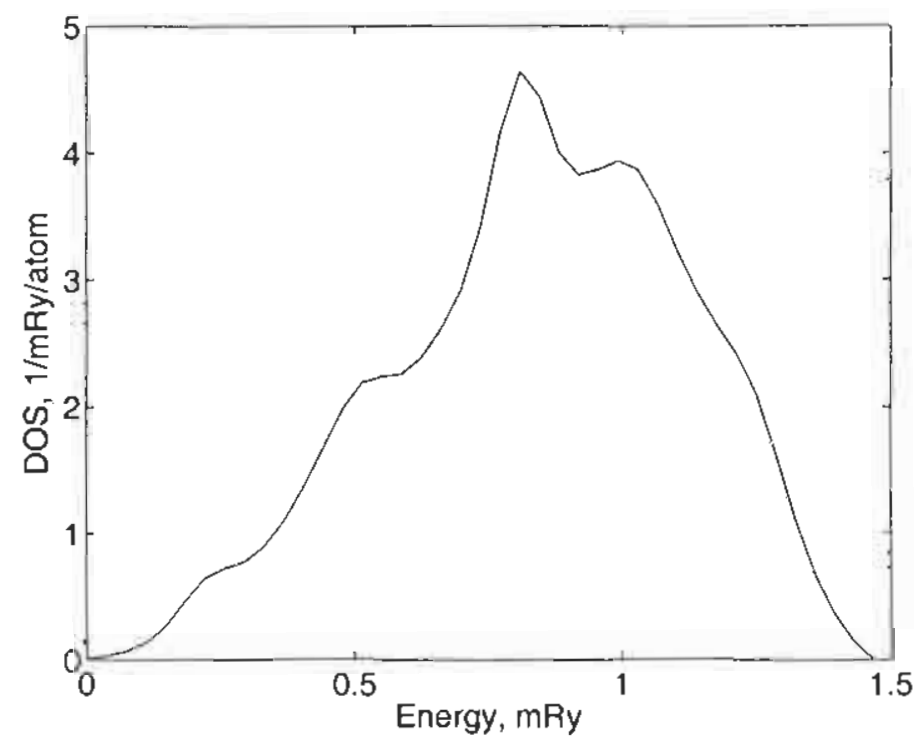

FIG. 1: Phononic density of states at $V / V_{0}=1.0$

In the Fig. 1 the phonon density of states at $V / V_{0}=1.0$ is prosented. Two maximums in the DOS are scen at 0.8 and $1.0 \mathrm{mRy}$. Apparently, they are connected with the optical branches of phonon spectrum and with the high energy acoustical branches. Also, it. is necessary to point out the snall feature at $0.25 \mathrm{~m} R \mathrm{y}$. This feature can be connected with softening of a whole phonon spectrum at low temperatures (charge density wave (CDW) transition). ${ }^{8}$ However, as it was indicated in ref. [8], a single soft mode related with CDW transition would involve a volume in reciprocal space too small to be observed in the phonon DOS.

DOS is vory fast varying function of both the energy and the volume. That is why the direct differentiation of it with respect to the volume (or pressure) is not well defined. Derivatives of some integral values, which depend on the phononic DOS, are much more informative.

In this respcct, it costs to say that the vibrational contribution to the thermodynamic functions of $U$ depends little on the delails of the phonon spectra. As an example, on the Fig.2 the dependance of the specific heat $C_{V}$ on the temperature calculated with the phonon DOS:

$$
C_{V}(T)=\frac{1}{k_{B} T} \int_{0}^{\infty} e^{\frac{\omega}{k_{G} T}}\left(\frac{\omega}{e^{\frac{\omega}{k_{B} B^{T}}}-1}\right)^{2} g(\omega) d \omega,
$$




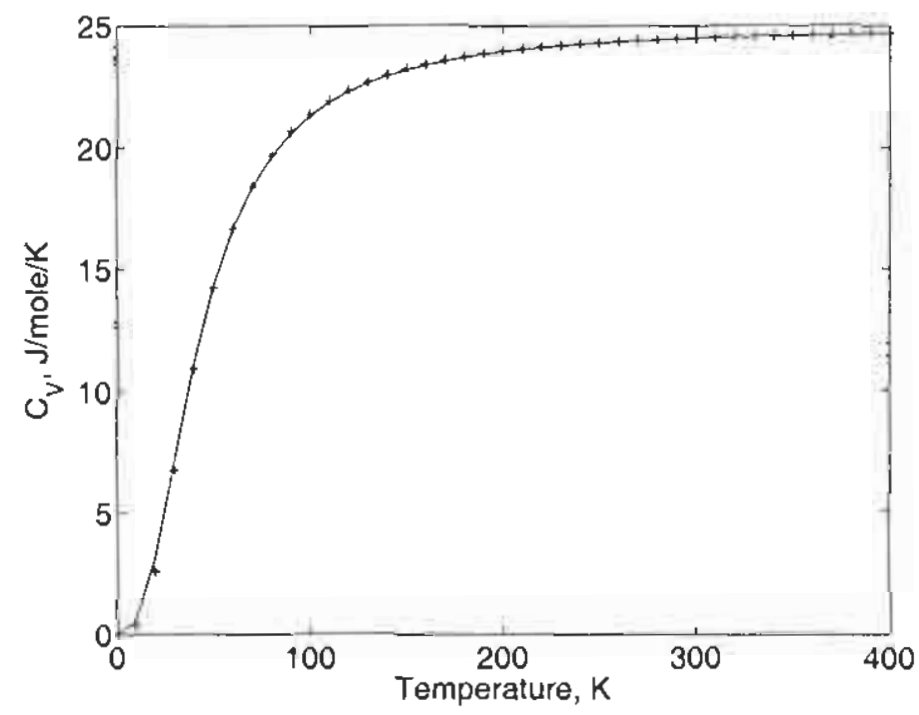

FIG. 2: Specific heat $C_{V}$ of $\alpha$-U versus temperature at equilibrium volume. Crosses are the results obtained with Debye model. Solid line - from the phonon DOS.

and with the Debye model:

$$
C_{V}(T)=9 k_{B} N_{A}\left(4 \frac{T^{3}}{\Theta_{D}^{3}} \int_{0}^{\Theta_{P}} \frac{x^{3}}{e^{x}-1} d x-\frac{\Theta_{D}}{T\left(e^{\frac{\Theta_{P}}{T}}-1\right)}\right),
$$

are presented. Debye temperature $\Theta_{D}$ has been calculated from the phonon DOS and the equality

$$
\frac{1}{2} \sum_{\mathrm{g} r} \omega_{\mathrm{g} a}=\frac{9}{8} N_{A} k_{B} \Theta_{D}
$$

where $N_{A}=2$ is the number of atoms in the unit cell. As it can be secn, two curves arc practically coincide. Small difference exists only at very low temperatures.

Thus, to obtain the volume dependance of the vibrational contribution to the thermodynamic functions of uanium, Debye temperatures have bocn found with using (4) at two more volumes $\left(V / V_{0}=0.95\right.$ and 0.90$)$. They are presented in the table I in comparison with the Debyc temperatures found from the bulk modulus via the semiempirical relation (9a) from the ref.[13]. As it can be seen, there is a closc coincidence. We think, however, that this is by chance, because bulk modulus $B$ have been calculated in GGA, but $g(\omega)$ - in LDA. It. seems, that the mutual cancellation of errors took place in the Debye temperaturc calculations from phonon DOS. First error is connected with the fact, that at $V / V_{0}=1.0\left(V_{0}\right.$ is the 
TABLE I: Comparison of the Debay temperature obtained from bulk modulus and from phonon DOS

\begin{tabular}{cccc}
\hline \hline$V / V_{0}$ & 1.00 & 0.95 & 0.90 \\
\hline$\Theta_{D}$ from $B$ & 176 & 190 & 217 \\
$\Theta_{D}$ from $g(\omega)$ & 178 & 188 & 214 \\
\hline
\end{tabular}

experimental equilibrium volume) the pressure is negative in LDA and correspondingly the phonon spectrum is softer. Second error is that the LDA itself gives the elastic properties of solid which are harder than they are in GGA. This second error is opposite to the first one.

Further, the Debye temperatures found from the phoron DOS were approximated by the relation

$$
\Theta_{D}(V)=\Theta_{0} e^{\gamma\left(\mathrm{I}-V / v_{0}\right)}
$$

where $\Theta_{0}=178 \mathrm{~K}$ is the Debye temperature at $V / V_{0}=1.0$ and $\gamma=1.6922$.

The derivative of $\Theta_{D}$ with respect to pressure can be calculated as

$$
\frac{d \Theta_{D}}{d P}=\frac{d \Theta_{D}}{d \frac{V}{V_{0}}} \frac{d \frac{V}{V_{0}}}{d P}=\gamma_{\gamma} \Theta_{D} \frac{V}{V_{0} B}
$$

where $B$ - bulk modulus.

Numerical value of $\frac{d \Theta_{D}}{d P}$ at $V / V_{0}=1.0$ is $0.223 \mathrm{~K} / \mathrm{hbar}$.

To assess the accuracy of separating the phonon and electron excitations, the specific heat $C_{F}$ of uranium has been calculated as a function of temperaturc and it has been compared with the experimental one. Debye model and the represcntation (5) have been used for the phonon contribution to the specific heat. The specific heat $C_{P}$ has been calculated from the relation

$$
C_{p}=C_{V}\left[1+\frac{T V C_{V}}{B \Theta_{D}^{2}}\left(\frac{d \Theta_{D}}{d V}\right)^{2}\right]
$$

For the electronic contribution, the representation from the first report upon the contract $^{12}$ was used. 


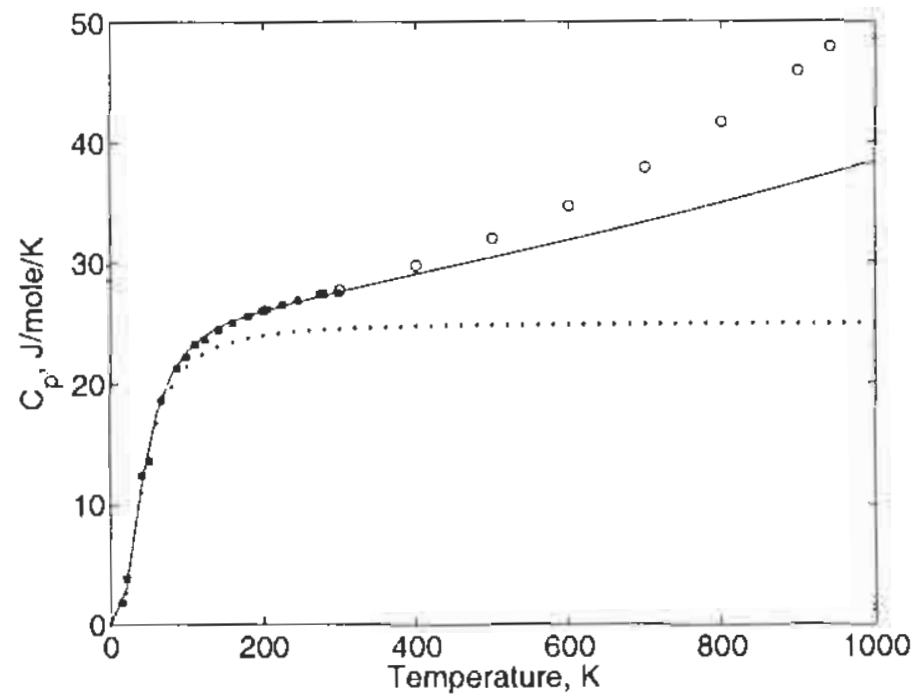

FIG. 3: Specific heat of $\alpha-U$ versus tomperature as calculated at zero pressure in comparison with the experimental data. Points - only phonon contribution. Solid line - phonon and electron contributions. Circles and astcrisks - experimental data.

As it is seen from a figure 3 , when we use only phonon contribution it allows us to describe $C_{F}$ well up to $100 \mathrm{~K}$ only. The addition of the electronic excitations describes $C_{P}$ up to $350 \mathrm{~K}$. But at higher tcmperatures, however, the disagreement increases. It can be proposed that. this is because anharmonicity of viblations of a lattice. However, in the light of the work [8], it is more probably that this is connected with a softening of a harmonic crystal, i.e. the temperature alters the electronic structure sufficiently to change the lattice dynamics. If so, the theoretical description of high-temperature thermodynamics of uranium based on the separation of the electronic and the phononic degrees of freedom, and on the electronic structure calculated at $\mathrm{T}=0 \mathrm{~K}$ must be replaced with mole sophisticated approach.

1 E. S. Fisher and H. J. McSkimin, J. of Appl. Phys, 29, 1473 (1958).

2 H. J. MeSkimin and E. S. Fisher, J. of Appl. Phys. 31, 1627 (1960).

3 E. S. Fisher and D. Dever, Phys, Rev. 170, 607 (1968).

4 S. van Sinaalen and T. F. George, Phys. Rov, B 35, 7939 (1987).

L. Fast, O. Eriksson, B. Johansson, J. M. Wills, G. Straub, H. Rooder, and L. Nordstrom, 
Phys. Rev. Lctt. 81, 2978 (1998).

6 J. Akella, S. Weir, J. M. Wills, and P. Söderlind, J. Phys.: Condens. Matter 9, L549 (1997).

7 W. P. Crummett, H. G. Smith, R. M. nicklow, and N. Wakabayashi, Phys. Rov. B 19, 6028 (1979).

8 M. E. Manley, B. Fultz, R. J. McQueeney, C. M. Brown, W. L. Hults, J. L. Smith, D. J. Thoma, R. Osborn, and J. L. Robertson, Phys. Rev. Lett. 86, 3076 (2001).

9 A. Kutepov and S. Kutepova, J. Phys.: Condens. Matter 15, 2607 (2003).

10 A. .L. Kutepov, Third report on the contract No.B530324.

II P. E. Blöchl, O. Jepsen, aııd O. K. Andersen, Phys. Rev. B 49, 16223 (1994).

12 A. .L. Kutepov, First report on the contract No.B530324.

13 P. Söderlind, L. Nordström, L. Yongming, and B. Johansson, Phys. Rev. B 42, 4544 (1990). 